\title{
Preventive role of low-molecular-weight heparin in unexplained recurrent pregnancy loss
}

\author{
E S Khan, ${ }^{1}$ MBBS, FCPS (O\&G); A Basharat, ${ }^{2}$ MBBS, FCPS (O\&G); M Jamil, ${ }^{3}$ MBBS, FCPS (O\&G); \\ S Ayub, ${ }^{4}$ MBBS, FCPS (O\&G); M A Khan, ${ }^{5}$ MBBS, MSc, FCPS (Nuclear Med) \\ ${ }^{1}$ Department of Obstetrics and Gynecology, Agha Khan University, Karachi, Pakistan \\ ${ }^{2}$ Department of Obstetrics and Gynecology, Alnafees Medical College, ISRA University, Islamabad, Pakistan \\ ${ }^{3}$ Department of Obstetrics and Gynecology, Shifa College of Medicine, Islamabad, Pakistan \\ ${ }^{4}$ Department of Pathology, Rawalpindi Medical College, Rawalpindi, Pakistan \\ ${ }^{5}$ Department of Nuclear Medicine, Nuclear Medicine, Oncology and Radiotherapy Institute (NORI), Islamabad, Pakistan
}

\section{Corresponding author: M A Khan (draleemkhan@yahoo.com)}

Background. Recurrent pregnancy loss (RPL) is a source of great distress for couples, and the search continues for an intervention to improve live birth rates in affected women. A daily injection of low-molecular-weight heparin (LMWH) is often prescribed to women with unexplained RPL, although evidence suggesting a benefit is limited.

Objective. To compare the efficacy of LMWH with a placebo in terms of live birth rates in women with unexplained RPL.

Methods. All pregnant females between 18 and 44 years of age who reported at the unit of obstetrics and gynaecology, Shifa International Hospital, Islamabad, during April 2013 to January 2014, who had a history of $\geq 2$ consecutive first trimester pregnancy losses were enrolled. All participants were randomly allocated to one of two groups. Group A received a daily dose of 40 mg enoxaparin (LMWH) subcutaneously and group B women received a placebo in the form of multivitamin tablets. Efficacy was defined in terms of live births after the age of viability (i.e. 24 weeks' gestation) and was compared in both treatment and control groups. Risk estimation was also performed and relative risk (RR) along with $95 \%$ confidence interval (CI) was calculated.

Results. The groups were similar in terms of mean age, gestational age and body mass index. Our results showed no statistically significant difference in live birth rates between the two groups, with $78.8 \%$ and $73.8 \%$ for group A and B, respectively ( $p=0.0574)$. A RR of 1.07 (95\% CI 0.9 - 1.3) was calculated for group A.

Conclusion. Subcutaneous enoxaparin in a once daily dose of $40 \mathrm{mg}$ did not improve the chance of live births in nonthrombophilic women with unexplained RPL when compared with the placebo.

S Afr J Obstet Gynaecol 2017;23(1):17-19. DOI: 10.7196/SAJOG.2017.v23i1.1112

Recurrent pregnancy loss (RPL) is one of the commonly encountered complications in reproductive medicine, as the aetiology is often unknown and there are few evidence-based diagnostic and treatment strategies available. There are varying definitions of RPL reported that include $\geq 2$ failed clinical pregnancies as documented by ultrasonography or histopathological examination; ${ }^{[1]}$ or 3 consecutive pregnancy losses, which are not required to be intrauterine. ${ }^{[2]}$ The definition of RPL also includes non-visualised pregnancy losses (biochemical pregnancy losses and/or pregnancies of unknown location) as they have the same negative impact on future live birth rate as intrauterine pregnancy losses. ${ }^{[3]}$ The European Society of Human Reproduction and Embryology released a 2014 consensus statement proposing that RPL describes repeated pregnancy loss regardless of anatomic location. They did not recommend the number of losses required for the problem to be defined as recurrent. However, the statement advised researchers and clinicians to clearly describe the type of pregnancy loss, gestational age, number of prior pregnancy losses and relevant details of ultrasound measurements. ${ }^{[4]}$

There is a consensus that healthy women should not undergo extensive evaluation after a single first trimester or early second trimester spontaneous miscarriage (up to 20 weeks), as these are relatively common sporadic events that occur in $\sim 10-15 \%$ of clinically recognised pregnancies under 20 weeks' gestation. The overall risk of miscarriage in the next pregnancy remains about $15 \%$ after one miscarriage, but rises to $17-31 \%$ after two consecutive miscarriages and to $25-46 \%$ after three miscarriages. ${ }^{[5]}$ Based on these and similar data, usual clinical practice is to initiate evaluation and treatment of RPL after two consecutive miscarriages. ${ }^{[5]}$ RPL remains unexplained in $\sim 50 \%$ of couples after evaluation; however, the chance of a live birth is good $(>50 \%)$ with no intervention and this must be considered in evaluating therapies for unexplained RPL. ${ }^{[6]}$ Although women with a history of RPL who become pregnant may be at a higher risk for developing fetal growth restriction and premature delivery, the detection of fetal cardiac activity in early pregnancy is reassuring of subsequent viable delivery. ${ }^{[7]}$

A variety of treatments have been offered to couples with unexplained RPL, including lifestyle modification, progesterone therapy, human menopausal gonadotropin, in vitro fertilisation and preimplantation genetic diagnosis. ${ }^{[8]}$ Anticoagulants have been studied in this context for a number of years. Different thrombophilia polymorphisms have been identified with RPL and intervention with thromboprophylaxis as preventive therapy has been proposed. 
However, the evidence is still insufficient for routine clinical use of anticoagulants in such women. ${ }^{[9]}$

RPL is a source of great distress for couples and a search for an intervention that could result in improved live birth rates in these women is ongoing. Therapeutic recommendations are largely based on clinical experience and data from observational studies. The objective of this study was to compare the efficacy of lowmolecular-weight heparin (LMWH) with a placebo in women with unexplained RPL, to generate evidence-based therapeutic recommendations for these patients.

\section{Methods}

This randomised controlled trial was carried out at the Obstetrics and Gynaecology Department of Shifa International Hospital, Islamabad, Pakistan, between April 2013 and January 2014. The study was approved by the hospital ethics committee and a fully informed consent was obtained from participants. During this period, females between 18 and 44 years of age who had a history of $\geq 2$ consecutive first trimester pregnancy losses were enrolled and assessed with adequate history, thorough clinical examination and necessary investigations. Pregnancy was confirmed by the quantitative beta human chorionic gonadotropin ( $\beta \mathrm{HCG}$ ) test and by ultrasound confirming fetal heart activity. Patients who presented with systemic lupus erythematosus, positive IgG and IgM anticardiolipin antibodies, positive for thrombophilia screening, any platelet function abnormality or a previous thromboembolic event requiring anticoagulant therapy (including heparin, aspirin or warfarin) and having sensitivity to acetylsalicylic acid, heparin or warfarin obtained by self-report, were excluded from the study. Patients who had any genetic cause (identified by karyotype analysis of both partners), anatomical (identified on hysterosalpingogram or sonohysterogram) or hormonal cause (identified by mid-luteal phase evaluation of progesterone, prolactin and thyroid-stimulating hormone) of RPL were also excluded from the study. All the patients were randomly allocated to one of two groups by the sealed envelope method. Group A women received a daily dose of $40 \mathrm{mg}$ LMWH subcutaneously and group B women received the placebo in the form of multivitamin tablets. Women in both groups were evaluated in the antenatal clinic every 6 weeks until delivery or pregnancy loss. Efficacy was defined in terms of live births after the age of viability, i.e. 24 weeks' gestation and was compared in both treatment and control groups by employing a $\chi^{2}$ test. A $p$-value of $<0.05$ was considered as statistically significant. Risk estimation was also performed and relative risk (RR) with a 95\% confidence interval (CI) was calculated.

\section{Results}

A total of 160 pregnant women were enrolled and equally dis-tributed into groups A (LMWH) and B (placebo). The groups were similar in terms of mean age, gestational age and body mass index (BMI) (Table 1). In group $A, 78.8 \%(n=63)$ completed the pregnancy with successful live births, while the percentage was slightly lower at $73.8 \%(n=59)$ in group B. However, the difference was not statistically significant ( $p=0.574$ ) (Table 2). An RR of 1.07 (CI 0.9 - 1.3) was calculated for the live birth rate of $78.8 \%$ in group A (Table 3 ).

\section{Discussion}

RPL affects $1-2 \%$ of women. In $>50 \%$ of such cases the cause remains unknown. High-quality data on the management of RPL are limited and reported studies on the aetiology, evaluation
Table 1. Demographic characteristics of groups A and B $(N=160)$

\begin{tabular}{lll}
\hline Variables, mean (SD) & A (LMWH) & B (placebo) \\
\hline Age (years) & $25.9(4.6)$ & $26.0(3.4)$ \\
Gestational age at enrolment (weeks) & $8.2(0.5)$ & $8.1(0.3)$ \\
BMI $\left(\mathrm{kg} / \mathrm{m}^{2}\right)$ & $29.9(3.5)$ & $29.1(2.7)$
\end{tabular}

Table 2. Comparison of efficacy in terms of live births between both groups $(N=160)$

\begin{tabular}{llll}
\hline & \multicolumn{3}{c}{ Live birth, $\boldsymbol{n}(\%)$} \\
\cline { 2 - 4 } Group & Yes & No & p-value \\
\hline A $(n=80)$ & $63(78.8)$ & $17(21.3)$ & \\
B $(n=80)$ & $59(73.8))$ & $21(26.3)$ & \\
Total & $122(76.3)$ & $38(23.7)$ & 0.574
\end{tabular}

Table 3. Risk estimates

\begin{tabular}{lll}
\hline Estimates & Value & $\mathbf{9 5 \%}$ CI \\
\hline OR (for group A/B) & 1.319 & $0.635-2.741$ \\
RR (for live birth = yes) & 1.068 & $0.898-1.270$ \\
RR (for live birth = no) & 0.810 & $0.463-1.416$
\end{tabular}

and management of RPL are mostly observational. For these reasons, therapeutic recommendations are largely based on clinical experience and data from these observational studies. ${ }^{[10]}$ Nevertheless, the prognosis for a successful future pregnancy is generally good: the overall live birth rates after normal and abnormal diagnostic evaluations for RPL are $77 \%$ and $71 \%$, respectively. ${ }^{[1-3]}$

Thrombosis of spiral arteries and the intervillous space on the maternal side of the placenta can impair adequate placental perfusion. The resulting abnormalities of the uteroplacental circulation may cause late fetal loss, intrauterine growth restriction, placental abruption or preeclampsia. A relationship with early pregnancy loss is less clear and may be restricted to specific thrombophilic defects; literature on the association between maternal inherited thrombophilia and RPL occurring in the first trimester is contradictory. A systematic review of the association between fibrinolytic defects and RPL found a significant association for factor XII deficiency (odds ratio (OR) 18.11, CI 5.52 - 59.4). ${ }^{[11]}$ Procoagulant microparticles may also contribute to the hypercoagulable state in these women and thus may interfere with successful implantation and fetal growth. ${ }^{[12]}$

Thrombophilia has been identified in $\sim 50 \%$ of women with RPL and thromboprophylaxis has been suggested as a treatment option. ${ }^{[1,2]}$ It has been reported in the literature that anticoagulation of women with certain inherited thrombophilias may improve maternal outcome (e.g. prevention of venous thromboembolism), but does not appear to prevent pregnancy loss. ${ }^{[13]}$ We explored the effects of LMWH (subcutaneous enoxaparin) in our settings. The role of LMWH in preventing pregnancy losses is based on the principle that placental circulation can become compromised in women with underlying prothrombotic tendencies, and administration of LMWH may be able to reduce this tendency, resulting in better fetal outcomes. 
In our study, the live birth rate in the placebo group was slightly lower than in the intervention group ( $73.8 \%$ v. $78.8 \%$, respectively). However, the difference was not statistically significant $(p=0.574)$. Our results are similar to those in studies reported in the literature. In a similar study, Pasquier et al..$^{[14]}$ enrolled 258 pregnant women with a history of unexplained recurrent miscarriage $(\geq 2$ consecutive miscarriages before 15 weeks' gestation) and a negative thrombophilia screen. They were randomly assigned to receive one daily subcutaneous injection of $40 \mathrm{mg}$ enoxaparin or placebo until 35 weeks' gestation. Their results indicated that $66.6 \%$ of the women who received enoxaparin had a live birth v. $72.9 \%$ of women who received the placebo $(p>0.05)$. They concluded that enoxaparin ( $40 \mathrm{mg}$ once daily) did not improve the chance of a live birth in nonthrombophilic women with unexplained RPL. ${ }^{[14]}$ In another similar multicentre, randomised controlled trial, Schleussner et al. ${ }^{[15]}$ determined whether LMWH increases live birth rates in women with unexplained RPL. They enrolled 449 women, with at least 2 early or 1 late miscarriages, at 5 - 8 weeks' gestation after confirmation of a viable pregnancy by ultrasonography. Women in the control group received multivitamin tablets, and the intervention group received vitamins and $5000 \mathrm{IU}$ of dalteparin-sodium for up to 24 weeks' gestation. The live birth rates were $86.0 \%$ and $86.7 \%$ in the intervention and control groups, respectively. They concluded that daily LMWH injections did not increase ongoing pregnancy or live birthrates in women with unexplained RPL. A 2014 Cochrane review including nine trials reported similar results, after evaluating the effects of either LMWH or aspirin, or a combination of the two, on women with RPL or without inherited thrombophilia. The authors found no evidence of an increased frequency of live birth among both groups. ${ }^{[16]}$ Visser et al. ${ }^{[17]}$ in their randomised, double-blind, multicentre trial, randomly allocated 207 women with $\geq 3$ consecutive first trimester miscarriages into one of three groups: $40 \mathrm{mg}$ enoxaparin and placebo $(n=68), 40 \mathrm{mg}$ enoxaparin and $100 \mathrm{mg}$ aspirin $(n=63)$ or $100 \mathrm{mg}$ aspirin $(n=76)$. They found a live birth rate of $71 \%$ (RR 1.17; CI 0.92 - 1.48) for enoxaparin and placebo and 65\% (RR 1.08; CI 0.83 - 1.39) for enoxaparin and aspirin when compared with aspirin alone (61\%). The difference was not statistically significant.

In summary, based on the present study results and other similar studies reported in the literature, the evidence is still not sufficient to recommend routine use of anticoagulants in women with unexplained RPL without inherited thrombophilia. Therapeutic intervention for RPL is guided by the underlying cause, and in all cases emotional support is important in caring for these often-anxious couples, which may enhance therapeutic success. There are limitations in the present study: firstly, placebo injections were not used and secondly, the sample size was relatively small.

\section{Conclusion}

Subcutaneous enoxaparin in a dose of $40 \mathrm{mg}$ once daily did not improve the chance of live births in non-thrombophilic women with unexplained recurrent pregnancy loss when compared with the placebo.

1. Practice Committee of American Society for Reproductive Medicine. Definitions of infertility and recurrent pregnancy loss: A committee opinion. Fertil Steril 2013;99(1):63. https://doi. org/10.1016/j.fertnstert.2012.09.023

2. Jauniaux E, Farquharson RG, Christiansen OB, Exalto N. Evidence-based guidelines for the investigation and medical treatment of recurrent miscarriage. Hum Reprod 2006;21(9):2216-2222 https://doi.org/10.1093/humrep/del150

3. Kolte AM, van Oppenraaij RH, Quenby S, et al. Non-visualized pregnancy losses are prognostically important for unexplained recurrent miscarriage. Hum Reprod 2014;29(5):931-937. https://doi. important for unexplained recil

4. Kolte AM, Bernardi LA, Christiansen OB, et al. Terminology for pregnancy loss prior to viability A consensus statement from the ESHRE early pregnancy special interest group. Hum Reprod 2015;30:495-498. https://doi.org/10.1093/humrep/deu29

5. Jaslow CR, Carney JL, Kutteh WH. Diagnostic factors identified in 1020 women with two versus three or more recurrent pregnancy losses. Fertil Steril 2010;93(4):1234-1243. https://doi org/10.1016/j.fertnstert.2009.01.166

6. Practice Committee of the American Society for Reproductive Medicine. Current clinical irrelevance of luteal phase deficiency: A committee opinion. Fertil Steril 2015;103(4):e27-e32. https://doi.org/10.1016/j.fertnstert.2014.12.128

7. Field K, Murphy DJ. Perinatal outcomes in a subsequent pregnancy among women who have experienced recurrent miscarriage: A retrospective cohort study. Hum Reprod 2015;30(5):1239experienced recurrent miscarriage: A retrosp
1245. https://doi.org/10.1093/humrep/dev044

8. Coomarasamy A, Williams H, Truchanowicz E, et al. A randomized trial of progesterone in women with recurrent miscarriages. N Engl J Med 2015;373(22):2141-2148. https://doi.org/10.1056/ nejmoal504927

9. Mumford SL, Silver RM, Sjaarda LA, et al. Expanded findings from a randomized controlled trial of preconception low-dose aspirin and pregnancy loss. Hum Reprod 2016;31(3):657-665. https:/ doi.org/10.1093/humrep/dev329

10. Christiansen OB, Nybo Andersen AM, Bosch E, et al. Evidence-based investigations and treatments of recurrent pregnancy loss. Fertil Steril 2005;83(4):821-839. https://doi.org/10.1016/j. fertnstert.2004.12.018

11. Sotiriadis A, Makrigiannakis A, Stefos T. Fibrinolytic defects and recurrent miscarriage: A systematic review and meta-analysis. Obstet Gynecol 2007;109(5):1146-1155. https://doi. org/10.1097/01.aog.0000260873.94196.d6

12. Laude I, Rongières-Bertrand C, Boyer-Neumann C. Circulating procoagulant microparticles in women with unexplained pregnancy loss: A new insight. Thromb Haemost 2001;85(1):18-21.

13. Abheiden C, Van Hoorn ME, Hague WM, et al. Does low-molecular-weight heparin influence fetal growth or uterine and umbilical arterial Doppler in women with a history of early-onset uteroplacental insufficiency and an inheritable thrombophilia? Secondary randomised controlled trial results. BJOG 2016;123(5):797-805. https://doi.org/10.1111/1471-0528.13421

14. Pasquier E, de Saint Martin L, Bohec C, et al. Enoxaparin for prevention of unexplained recurrent miscarriage: A multicenter randomized double-blind placebo-controlled trial. Blood 2015;125(14):2200-2205. https://doi.org/10.1182/blood-2014-11-610857

15. Schleussner E, Kamin G, Seliger G, et al. Low-molecular-weight heparin for women with unexplained recurrent pregnancy loss: A multicenter trial with a minimization randomization scheme. Ann Intern Med 2015;162(9):601-609. https://doi.org/10.7326/m14-2062

16. De Jong PG, Kaandorp S, Di Nisio M, et al. Aspirin and/or heparin for women with unexplained recurrent miscarriage with or without inherited thrombophilia. Cochrane Database Syst Rev 2014;CD004734. https://doi.org/10.1002/14651858.cd004734.pub4

17. Visser J, Ulander VM, Helmerhorst FM, et al. Thromboprophylaxis for recurrent miscarriage in women with or without thrombophilia. HABENOX: A randomized multicentre trial. Thromb Haemost 2011;105(2):295-301. https://doi.org/10.1160/th10-05-0334 